\title{
LAS EMPRESAS DE ALIMENTOS DE MANIZALES GENERAN GANANCIAS PERO DESTRUYEN VALOR ECONÓMICO AGREGADO (EVA)
}

\author{
FOOD COMPANIES IN MANIZALES \\ GENERATE PROFITS BUT \\ DESTROY THE ECONOMIC VALUE ADDED (EVA)
}

\author{
Gabriel Eduardo Escobar Arias ${ }^{1}$ \\ Docente Universidad Autónoma de Manizales \\ Grupo de Investigación en Empresariado
}

Palabras Clave: Valor económico agregado, Costo de capital, Rentabilidad del activo, Estados financieros.

Keywords: Economic value added, capital cost, profitability on assets, financial statements

\section{Resumen}

Este artículo es producto de la investigación “Medición del Valor Económico Agregado para las Principales Empresas de Alimentos de la Ciudad de Manizales" perteneciente a la línea de Finanzas del grupo de investigación en Empresariado, de la Facultad de Estudios Sociales y Empresariales de la Universidad Autónoma de Manizales. Dicha investigación es llevada a cabo durante los años 2007 - 2008 y tiene en cuenta a 19 empresas de la ciudad. En él damos cuenta de cómo las empresas estudiadas de Manizales obtienen ganancias pero destruyen valor económico agregado, caso que, corregido, permitiría a las empresas ser más eficientes, efectivas y obtener mayores ganancias.

\section{Abstract}

This paper shows the results of research called "Measurement of the Economic Value Added for the Main Food Companies in Manizales," which belongs to the Finances line of investigacion that pertains to the research group Empresariado of the Faculty of Social and Business Studies of the Autonoma University of Manizales. This research was carried out between 2007 and 2008 and held in 19 local companies. The result of the study shows that these companies make profits but destroy the economic value added, which, if corrected, would permit companies be more efficient, effective and make higher profits ${ }^{2}$.

\footnotetext{
1 Economista. Especialista en finanzas. Profesor de la Universidad Autónoma de Manizales - UAM. Candidato a magíster en Administración financiera por la Universidad Tecnológica de Pereira.

2 Resúmenes traducidos por el gestor de traducciones del Instituto de Idiomas de la UAM, Carlos A. Muñoz Torres, Máster en Teoría de la Traducción, Universitat Autònoma de Barcelona. Resúmenes revisados por Kevin Guzzo, nativo del inglés, estudiante de la Maestría en Traducción de la Universidad Autónoma de Manizales.
}

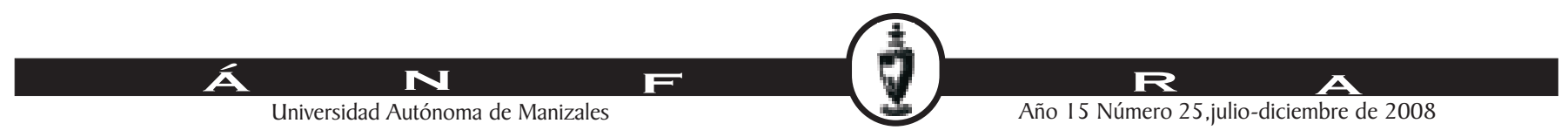




\section{Introducción}

Publicaciones, como la Revista Dinero, presentan, anualmente, un informe completo de las 5.000 empresas más grandes del país y uno de los indicadores para establecer este ranking es el Valor Económico Agregado (EVA). Este indicador permite a las empresas saber si son sostenibles o no; posibilita, además, medir la gestión en la toma de decisiones financieras y conocer el grado de rentabilidad de las mismas. Es por esto que este tema, se convierte, para el grupo de investigación en Empresariado, en un proyecto de investigación.

El artículo que presentamos a continuación es producto de la investigación "Medición del Valor Económico Agregado para las Principales Empresas de Alimentos de la Ciudad de Manizales" perteneciente a la línea de Finanzas del grupo de investigación en Empresariado, de la Facultad de Estudios Sociales y Empresariales de la Universidad Autónoma de Manizales. Dicha investigación es llevada a cabo durante los años 2007 2008 y tiene en cuenta a 19 empresas de la ciudad.

Nuestro propósito con la investigación, basados en la información de los estados financieros, reportados por las empresas a la Superintendencia de Sociedades, es calcular el EVA y con él determinar si las empresas estudiadas generaban o destruían valor.

Para lograr nuestro cometido, primero, desagregamos de esos estados financieros, las cuentas necesarias para poder calcular el EVA; luego, se calculó la rentabilidad operativa de esas empresas; en el tercer paso, se dedujo el costo de capital promedio ponderado, y por último, se obtuvo el Valor Económico Agregado en términos porcentuales y monetarios.

Presentamos a continuación, a los lectores, al público interesado y a los empresarios del sector de alimentos de Manizales los resultados de este trabajo que, por su utilidad, da origen a otras investigaciones para abarcar otros sectores económicos de la ciudad, con el fin de determinar, en el futuro próximo, el grado de competitividad de nuestra industria, objetivo de nuestra línea de investigación.

\section{Problema De Investigación}

En la Asignatura de Planeación Financiera, a mi cargo, se estudia como uno de los temas centrales el Valor Económico Agregado (EVA). En la clase, se realizan simulaciones, talleres, ejercicios donde se calcula el EVA y se hacen los respetivos análisis; como consecuencia, se genera la inquietud de aplicar estos conocimientos a la industria manizaleña, inquietud que es llevada al grupo de investigación y allí se decide realizar un estudio en las empresas de alimentos de Manizales.

Al comienzo se tienen ciertas inquietudes tales como, si las empresas generaban la suficiente información para calcular la rentabilidad del activo como indicador de operatividad; si la información recibida por la Superintendencia de Sociedades se encontraba debidamente clasificada; cómo depurar y clasificar la información que debía ser extraída del Banco de la República y del Departamento Nacional de Planeación para el cálculo del costo de capital promedio ponderado, etc.

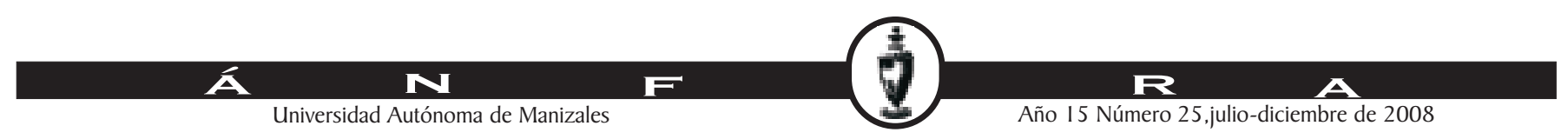


Todas estas inquietudes nos permiten enfocarnos en el problema principal que consistía en calcular el EVA a las empresas de alimentos de la ciudad de Manizales y resolver la pregunta que nos hacíamos sobre si las empresas son generadoras o destructoras de valor. Podemos concretar la pregunta de la siguiente forma: ¿Son las empresas de alimentos de la ciudad de Manizales generadoras o destructoras de Valor?

\section{Método.}

Para llevar a cabo esta investigación se realizaron los siguientes pasos:

? Se consiguieron, desde la Superintendencia de Sociedades, los estados financieros de las empresas más grandes productoras de alimentos (19 en total)

? Una vez obtenidos dichos estados, se procede a depurar la información y a desagregar esos estados financieros en: activos corrientes operativos, activos fijos operacionales, otros activos operativos, pasivos operativos de corto y largo plazo, patrimonio, ingresos, costos y gastos operativos.

? Luego, calculamos la rentabilidad del activo y el costo de capital promedio ponderado

? Por último, realizamos el cálculo del EVA de cada una de las empresas objeto de la investigación

? Conclusiones.

\section{Referente Conceptual.}

Para el estudio realizado tuvimos en cuenta los siguientes conceptos, esenciales a la indagación.

3.1 Valor Económico Agregado. En su libro Valoración de Empresas, Gerencia del Valor y EVA, Oscar León García afirma: "Las empresas han acogido el término valor agregado como uno más de su lenguaje cotidiano. Valor agregado para los clientes, cadena de valor agregado, decisiones que agreguen valor, etc., son expresiones con las que ya se está familiarizado ${ }^{3 "}$ Tal vez, los empresarios de Manizales también usen este lenguaje, sin embargo, nuestra inquietud está en saber si estos dueños de empresas, aplican y hacen uso de estos conceptos.

El término valor agregado nace, hace varias décadas, en la empresa Stern Stewart \& Co., que es asesora empresarial al nivel mundial, lo define como una estimación del monto de las ganancias que difieren de la tasa de rentabilidad mínima requerida (contra inversiones de riesgo comparable) para los accionistas o prestamistas. La diferencia puede ser una escasez o exceso de rentabilidad. Quiere decir esto que la generación de valor está amarrada de forma directa con la rentabilidad operacional; es decir, en la medida en que la organización tenga la capacidad de generar una rentabilidad operativa suficiente para cubrir los costos de la operación, se genera valor agregado; por esta razón no es un modelo nuevo ni novedoso, sino una oportunidad para esta compañía de lanzar una marca al mercado EVA ${ }^{\oplus}$ y explotarla, en donde lo nuevo solamente es una comparación entre rentabilidad y costo de capital y el resultado es la generación o destrucción de valor.

En la actualidad, hay varios factores que obligan a tener en cuenta la generación de valor al interior de las organizaciones, estos son: el flujo mundial de capitales, el reto
3 GARCÍA. S. Oscar León.

Valoración de Empresas, Gerencia del Valor y EVA. Cali:

Prensa Moderna Impresores S.A. 2003, p 3 .

\section{A N Universidad Autónoma de Manizales


de la apertura de las economías y la necesidad de mejores medidas de evaluación del desempeño, veamos cada uno de estos a continuación:

Flujo mundial de capitales. Hoy vemos como un empresario o un inversionista o un especulador pueden adquirir títulos de renta fija o variable en cualquier lugar del mundo y en el momento que lo desee a través del uso de la Internet; esto hace también que conseguir recursos, para el empresario, sea aún más fácil a menores costos y de manera más rápida y eficiente, lo que causa que la competitividad empresarial se vea afectada en la medida que algunos empresarios participan de estos procesos y otros no. Aquel que sea participe tendrá el derecho a conseguir recursos en cualquier moneda aprovechando el tipo de cambio entre una y otra, a unas tasas de interés más bajas de forma más rápida y esto causa un incremento en la competitividad y en la generación de valor, en la medida que el costo de capital ${ }^{4}$ de la empresa sea más bajo.

El reto de la apertura de las economías. Es otro factor determinante para la competitividad y la generación de valor de las organizaciones; el empresario puede tomar dos caminos: uno, es el de continuar sus operaciones como lo ha venido haciendo sin tener en cuenta que la apertura es una oportunidad para acceder a nuevos mercados o para la importación de bienes de capital que permitan que sus procesos mejoren en estos aspectos: tiempo, calidad y costo. Dos, ingresando a este modelo económico en el que tiene acceso a mayor información, a nuevos mercados y a bienes de capital que facilitan su eficiencia operativa y pueda incrementar la generación de valor, producto de la mejora en las capacidades y las eficiencias que este tipo de decisiones trae hacia el interior de la organización.

Necesidad de mejores medidas de evaluación del desempeño. Las organizaciones necesitan de indicadores financieros que puedan revelar el desempeño de sus actividades, la generación de valor es uno de ellos. El empresario ya sea grande, mediano o pequeño, puede determinar mediante el uso del EVA cómo está en su actividad principal, es decir, en qué grado de eficiencia operativa se encuentra, si está destruyendo valor o por el contrario es un empresario que en sus operaciones apunta a la competitividad y a la generación de valor, producto de ejercer su actividad principal.

Otra definición para el EVA es, desde un punto de vista económico, "coste de los factores internos a la empresa, es decir, aportación de la empresa al proceso de producción de bienes o servicios como salarios, intereses, arrendamientos, impuestos, gastos financieros, seguros, etc. ${ }^{5}$ "Según lo anterior, se agregar valor mediante un proceso productivo eficiente de la organización y esto da como resultado un producto o servicio, para el cual se incurre en costos. No se debe confundir esta productividad con utilidad, puesto que ésta es un resultado del ejercicio, mientras que el valor agregado (productividad) es el resultado de la actividad después de deducir costos adicionales que la utilidad no tiene en cuenta sea ésta operativa o neta.

Oriol Amat nos ofrece otra definición en su libro Valor económico agregado EVA, este es:

"El importe que queda una vez que se han deducido de los ingresos la totalidad de los gastos, incluidos el costo de oportunidad del capital y los impuestos. Por tanto EVA considera la productividad de todos los factores utilizados para desarrollar la actividad empresarial. En otras palabras, el EVA es lo que queda una vez que se han tendido todos los costos y gastos y se ha satisfecho una rentabilidad mínima esperada por parte de los accionistas. En consecuencia, se crea valor en una empresa cuando la rentabilidad generada supera el costo de oportunidad de los accionistas ${ }^{6}$."
4 El costo de capital puede definirse como costo de financiación de una organización, también conocido como costo de capital promedio ponderado.

5 ANDERSON, Arthur. Diccionario de economía y negocios. Madrid: Espasa. 1999, pág. 679.

6 AMAT, Oriol. Valor económico agregado EVA Bogotá: Norma 1999, pág.. 32

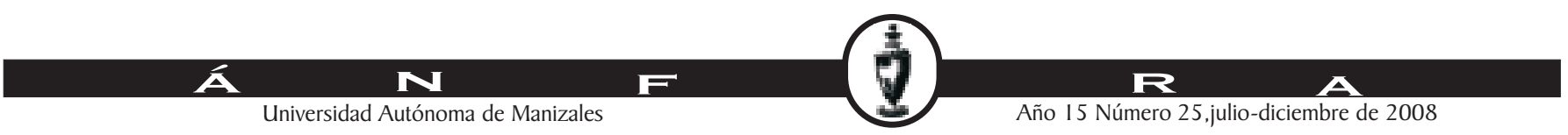


Hay que tener en cuenta entonces para el cálculo del EVA estos costos adicionales, que no incluye el estado de resultados de una empresa y como se mencionó en la definición anterior el costo de capital es un costo adicional que no tiene en cuenta este estado financiero. Hoy por hoy, muchas gerencias están enfocadas en medir sus resultados en la generación de utilidades; pero como se muestra, mediante la definición anterior, los resultados no deben ser observados a partir de este rubro sino más bien del indicador EVA como factor de competitividad financiero y no solamente financiero porque generar valor agregado significa una estrecha relación entre cada uno de los departamentos, que componen la organización donde cada uno aporta para el logro de este resultado cada vez que sea positivo, puesto que la generación de valor no es solamente un objetivo financiero sino un objetivo organizacional como lo apunta el autor Oscar León García la generación de valor agregado es una cultura de las organizaciones en todos sus sentidos y relaciones, ya sea con los clientes, empleados, proveedores, dueños, etc.

Generar valor no es simplemente un resultado financiero, es una estrategia empresarial como factor de competitividad donde el resultado de éste muestra el cumplimiento de los objetivos organizacionales como un todo y no de forma independiente o departamentalizada, la generación de valor se convirtió en una meta organizacional y no en una meta financiera.

Para el logro de cada objetivo organizacional, las cuentas operativas, que son las fuentes para la generación del valor, deben determinarse, es decir, encontrar los factores que la producen para así fijar un control sobre ellas con el fin de asegurar el logro de dichos objetivos. Al nivel financiero a estos se les conoce como los inductores del valor que son la rentabilidad del activo y el costo de capital.

El valor económico agregado EVA, se calcula mediante la siguiente fórmula:

$\mathrm{EVA}=$ Rentabilidad del activo - Costo de capital promedio ponderado

\section{EVA \% = ROI - C.C.P.P.}

Este arroja un resultado porcentual en términos efectivo anuales (E.A.) para el caso particular, en la medida que éste sea positivo, hay una generación de valor y si su resultado es negativo hay, por el contrario, destrucción de valor. Cuando el resultado porcentual es cero significa que no hay ni destrucción ni generación de valor.

El valor económico agregado EVA, también se puede medir en unidades monetarias, donde el EVA porcentual se multiplica por la cantidad de activos operativos así:

EVA $\$=($ Capital de trabajo + activos fijos operativos $) *$ EVA $\%$

\section{EVA \$ = Activos operativos - EVA \%}

De acuerdo a las fórmulas anteriores, se genera valor económico agregado EVA en la medida en que la rentabilidad del activo (ROI) sea mayor que el costo de capital promedio ponderado (C.C.P.P.), donde la estrategia empresarial es incrementar la rentabilidad y disminuir el costo de capital como se indicó anteriormente.

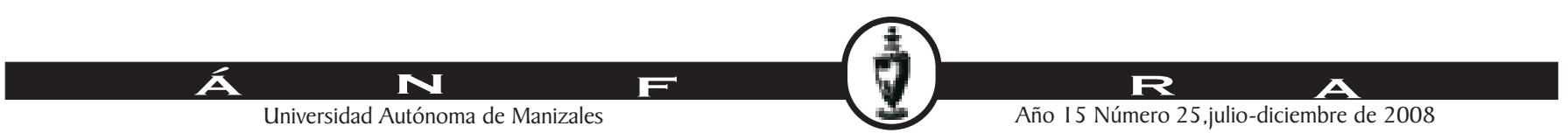


3.2 Sistema de creación de valor: Financieramente, la creación de valor en una organización se logra implementando procesos en los cuales se requiere de un monitoreo permanente y de la toma de decisiones por parte del empresario; para esto es importante:

? Definir cuáles son los indicadores que le dan información sobre los inductores de valor agregado.

? Gestionar para que se aplique cada uno de estos inductores de valor.

? Valorar la organización

? Monitoreo del valor.

Para definir los indicadores que le dan al empresario información sobre los inductores de valor, éste debe identificar claramente cuáles son las variables que afectan la actividad principal del negocio; es decir, cuales perjudican de forma directa la generación o destrucción del valor y cuáles no. Los estados financieros arrojan información útil para medir estas variables que son: el balance general, los activos operativos y aquellos que, de acuerdo a su uso, apuntan a que en la actividad principal se contribuya a la generación o destrucción de valor agregado. También el estado de resultados muestra información fundamental sobre la operatividad, es decir, da respuesta a los ingresos o egresos que arroja la información contable, producto de ejercer la actividad del negocio.

De la información contable se puede extraer aquella información que apunta hacía la generación de valor, de los activos se deben extraer aquellos que contribuyen a la generación de rentabilidad, producto de ejercer la actividad principal, siendo estos: los activos corrientes operativos, los activos fijos operativos y los otros activos operativos, a manera de ejemplo podemos nombrar:

? Activos corrientes operativos: Caja, bancos, cuentas por cobrar e inventarios.

? Activos fijos: Edificios, maquinaría y equipo.

? Otros activos: Patentes, gastos operativos pagados por anticipado.

En los pasivos operativos también encontramos de corto o de largo plazo, tales como: las obligaciones financieras, los proveedores, las obligaciones con los empleados, los impuestos, entre otros.

Las cuentas que conforman el patrimonio en su totalidad son operativas, algunas son: el capital, las utilidades y las reservas.

El estado de resultados, como estado financiero, también arroja información fundamental sobre la actividad principal del negocio como son: las ventas, los costos y los gastos operativos.

De toda la información anterior se puede extraer aquellos indicadores que son propios de la generación de valor y producto de la actividad principal, como son: la rentabilidad del activo, la rentabilidad del patrimonio y el costo de capital promedio ponderado.

Para la gestión de cada indicador, el administrador financiero debe estar muy pendiente de los estados contables, es decir, debe realizar permanentemente una revisión de cada una de las cuentas nombradas anteriormente, tanto del balance general, como del estado de resultados; esto debido a que, como lo mencionan algunos académicos, los estados financieros son una fotografía de la organización en una fecha determinada y muchas veces por mostrar mejores resultados, como ejemplo, un fin de mes o de año,

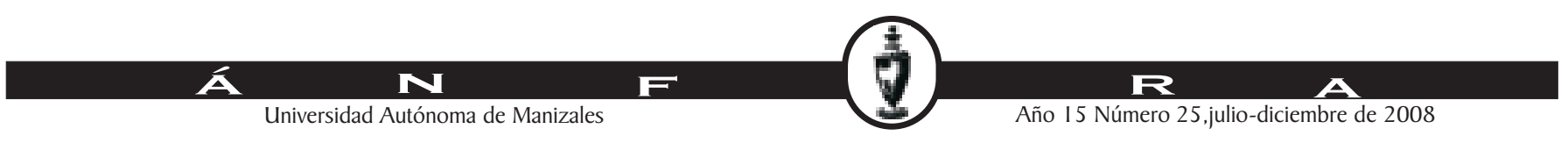


algunos administradores hacen ajustes para que estos estados financieros muestren mejores resultados.

3.3 Rentabilidad del activo: Es la ganancia que tiene la empresa por la correcta operación de sus activos operativos. Según Oscar León García en su libro Valoración de Empresas Gerencia del Valor y EVA "Es la rentabilidad que resulta de relacionar la utilidad operativa después de impuestos con los activos netos de operación" ${ }^{\prime \prime}$

La rentabilidad del activo es la resultante de dividir la Utilidad Operativa después de I mpuestos (UODI) entre los activos operativos:

\section{RENTABILIDAD DEL ACTIVO = UODI / ACTIVOS NETOS OPERATIVOS}

La utilidad operativa después de impuestos es la ganancia operacional después del pago del impuesto de renta de la actividad principal y los activos netos operativos son la sumatoria entre los activos corrientes operativos (caja, bancos, cuentas por cobrar e inventarios) y los activos fijos. Las cuentas operativas del activo que contribuyen a la generación de ingresos por ejercer la actividad principal son: caja, bancos, cuentas por cobrar e inventarios, estas cuentas corresponden a lo que en finanzas se denomina capital de trabajo que son los desembolsos o recursos monetarios necesarios, para la correcta operación de la empresa y generalmente están representados por la sumatoria entre cuentas por cobrar, inventarios y el disponible.

Las cuentas que hacen parte del análisis para el cálculo del capital de trabajo son:

$\begin{array}{ll}1105 & \text { Caja } \\ 1110 & \text { Bancos } \\ 1120 & \text { Cuentas de ahorro } \\ 1125 & \text { Fondos } \\ 1305 & \text { Clientes } \\ 1310 & \text { Cuentas corrientes comerciales } \\ 1330 & \text { Anticipos y avances } \\ 1345 & \text { Ingresos por cobrar } \\ 1405 & \text { Materias primas } \\ 1410 & \text { Productos en proceso } \\ 1430 & \text { Productos terminados } \\ 1435 & \text { Mercancías no fabricadas por la empresa } \\ 1460 & \text { Empaques y embases } \\ 1465 & \text { Inventarios en tránsito }\end{array}$

De acuerdo a lo anterior éstas serán las cuentas para el cálculo del capital de trabajo en las empresas productoras y comercializadoras de alimentos de la ciudad de Manizales.

Otro aspecto para tener en cuenta en el cálculo de la rentabilidad del activo son los activos fijos operativos, que son aquellos que se utilizan para ejercer la actividad principal del negocio entre estos tenemos:

$15 \quad$ Propiedad planta y equipo netos

Para el cálculo de la utilidad operativa, después de impuestos, en el balance general para cada caso aparece la utilidad operacional, que es la resultante de la diferencia
7 C f r. C a p it u 1 o 3 Macroinductores de valor: rentabilidad del activo pag.79

\section{A $\quad \mathbf{N}$ Universidad Autónoma de Manizales


entre los ingresos operativos y los costos y gastos operacionales; para el cálculo de la tasa de impuestos se trabajará con la tasa vigente para el periodo objeto de estudio, la cual asciende al $38,5 \%$ de las utilidades obtenidas durante el periodo.

3.4 Costo de Capital Promedio Ponderado (C.C.P.P.): Para el empresario es muy importante tener el conocimiento sobre el costo de capital, puesto que en toda evaluación económica y financiera se requiere tener una idea aproximada de los costos de las diferentes fuentes de financiamiento que utiliza la empresa para emprender sus proyectos de inversión. Además, el conocimiento del costo de capital y cómo es este influenciado por el apalancamiento financiero, permite tomar mejores decisiones en cuanto a la estructura financiera de la empresa. Finalmente, existe otro gran número de decisiones tales como: estrategias de crecimiento, arrendamientos y políticas de capital de trabajo, las cuales requieren del conocimiento del costo de capital de la empresa, para que los resultados obtenidos con tales decisiones sean acordes a las metas y objetivos que la organización ha establecido.

Para el C.C.P.P., existen algunas definiciones, entre las cuales podemos nombrar las siguientes:

? La tasa de interés que los inversionistas, tanto acreedores como propietarios, desean le sea pagada para conservar e incrementar sus inversiones en la empresa.

? Ponderado de las diferentes fuentes de financiamiento.

? La tasa de interés que iguala el valor presente de los flujos netos recibidos por la empresa, con el valor presente de los desembolsos esperados.

? El límite inferior de la tasa interna de rendimiento que un proyecto debe rendir para que se justifique el empleo del capital para adoptarlo.

Obviamente todas estas definiciones son equivalentes. Lo importante es desarrollar una metodología que determine el costo de cada una de las fuentes de financiamiento, tanto internas como externas.

Otra forma más de definir el concepto de costo de capital, es el costo de operar y obtener activos operativos para un correcto funcionamiento de la estructura organizacional.

\section{Cálculo De La Rentabilidad Del Activo:}

El cálculo de la rentabilidad del activo se realizará de forma individual para cada una de las empresas bases del estudio, de acuerdo a la base de datos las 19 empresas a estudiar son:

\section{NOMBRE}

Industrias Normandy Colombiana de Deshidratados S.A.

Panadería La Victoria

Central de Sacrificio Manizales S.A.

C.I. Super de Alimentos S. A.

Sociedad productos Lácteos de Caldas

Indiana S.A.

Central Lechera de Manizales

\section{NIT}

890807529

890807354

890807115

890806225

890805267

890805130

890804537

890800252

\section{A N} Universidad Autónoma de Manizales 
Industria de alimentos Gransoli y CIA.

Altopasti S.A.

H. M. Y CIA.

Suzette S.A.

Aristizabal Arango $\mathrm{Y} \mathrm{ClA}$.

Café Nevado Limitada

Incolfec S.A.

Deli Apa S.A.

Foodex S.A.

Descafeinadora Colombiana S.A.

Productora de Gelatina S.A.
830513638

810005055

810004572

810003259

810002322

810002193

810002085

800051756

800046958

800045228

860010192

Tabla 1. Cálculo de la rentabilidad del activo por empresa.

Tabla realizada por el autor donde se muestra de forma detallada la rentabilidad del activo por empresa objeto del estudio, determinantes para el cálculo del valor económico agregado.

\begin{tabular}{|l|l|}
\hline NOMBRE DE LA EMPRESA & RENTABILIDAD DEL ACTIVO \\
\hline Industrias Normandy & $9,33 \%$ \\
\hline Colombiana de Deshidratados S.A. & $9,16 \%$ \\
\hline Panadería la Victoria & $8,54 \%$ \\
\hline Central de sacrificio de Manizales S.A. & $12,12 \%$ \\
\hline C.I. Super de Alimentos S.A. & $7,02 \%$ \\
\hline Sociedad Productos Lácteos de Caldas & $0,57 \%$ \\
\hline Indiana S.A. & $-0,19 \%$ \\
\hline Central Lechera de Manizales & $3,77 \%$ \\
\hline Industria de Alimentos Gransoli & $1,09 \%$ \\
\hline Altopasti S.A. & $-13,07 \%$ \\
\hline H M y Cía. & $1,40 \%$ \\
\hline Suzette S.A. & $1,49 \%$ \\
\hline Aristizabal y Arango y Cía. & $-183,81 \%$ \\
\hline Café nevado LTDA. & $-9,52 \%$ \\
\hline Incolfec S.A. & $3,81 \%$ \\
\hline Deli Apa S.A. & $8,99 \%$ \\
\hline Foodex S.A. & $3,23 \%$ \\
\hline Descafeinadora Colombiana S.A. & $-0,46 \%$ \\
\hline Productora de Gelatina S.A. & $4,60 \%$ \\
\hline
\end{tabular}

De acuerdo a la información anterior podemos observar que la rentabilidad para la mayoría de empresas objeto del estudio es bastante reducida, inclusive, 4 de ellas presentan rentabilidad negativa, se pretendía mostrar una rentabilidad del activo promedio para las empresas de alimentos de la ciudad de Manizales, pero al mostrar datos tan dispersos un promedio no sería un dato representativo para la obtención de posibles conclusiones.

\section{Cálculo Del Costo De Capital Promedio Ponderado}

Para el cálculo del costo de capital se debe tener en cuenta solamente aquellas cuentas que son generadoras de costo, es decir, dentro del proceso de financiación de la organización algunas cuentas tanto del pasivo como del patrimonio generan un costo a

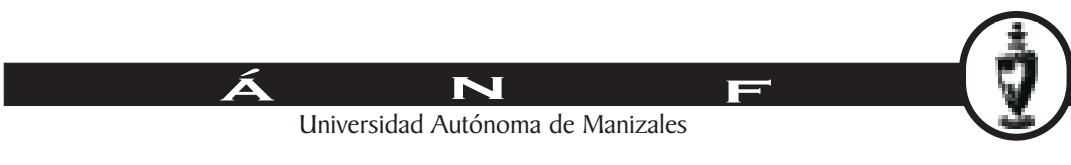


la hora de realizar dicha financiación, mientras que hay otras fuentes que son totalmente a cero costo, para el cálculo de este indicador, solamente se tendrán en cuenta las que generan este costo.

De acuerdo a la información obtenida, para efectos del cálculo del costo de capital se tomará la tasa promedio del sistema para el año, debido a que resulta imposible de definir el mes en el cual la empresa adquirió la obligación financiera, donde la tasa promedio de colocación para el sistema financiero Colombiano durante el años 2006, tanto para los créditos de largo como de corto plazo fue del: 11,51\% E. A.

Para el cálculo de la tasa mínima de rendimiento requerida por el inversionista (T.M.R.R.I.) se hace uso de la siguiente información:

La tasa promedio en títulos TES e Colombia para el año 2006 asciende al 9,05\% E. A. La tasa DTF para Colombia promedio durante el año 2006 ascendió al 6,27\% E.A. La tasa promedio para los bonos del tesoro de los Estados Unidos asciende al 4,87\% E.A. Durante el año 2006 en promedio la devaluación acumulada ascendió al 0,06\% E. A. El indicador beta, el cual maneja el riesgo sistémico sectorial ascendió dl 0,72 durante el año 2006.

Para el cálculo del riesgo del inversionista se trabajará con la tasa DTF más los puntos adicionales por riesgo (spread) que a criterio del autor en el sector de alimentos que no requiere de grandes inversiones en comparación con otras industrias, se trabajará con una tasa riesgo adicional equivalente al $2 \%$, lo que genera una tasa sectorial equivalente al 8,39\% E.A. (DTF=6,27\% E.A + Spread 2\% E.A).

Para el cálculo del riesgo país se utilizará el indicador Embi (Indicador de riesgo de mercados emergentes) durante el año 2006 este ascendió a 181 puntos básicos, que es equivalente el $1,81 \%$ E.A.

\subsection{Cálculo de la tasa mínima de rendimiento requerida por el inversionista}

(T.M.R.R.I.): La tasa mínima de rendimiento requerida se basa en el modelo CAPM (valoración de activos de capital) el cual establece que es el resultado de la suma de las tasas libre de riesgo y la tasa del mercado multiplicada por el riesgo sectorial:

$C A P M=R f+(R m-R F) \times B$

Como se mostró en párrafos anteriores para el caso Colombiano se propone un ajuste al modelo de tal manera que la mezcla de rendimientos y riesgos sea adecuada para el entorno en el cual se movilizan nuestras empresas. Donde la T.M.R.R.I adecuada para las empresas de alimentos en Colombia es: (Tabla 2)

T.M.R.R.I. $=(9,05 \%+6,27 \%+4,87 \%)+(1,81 \%+0,06 \%+8,39 \%) \times 0,72$

\section{T.M.R.R.I. $=27,57 \% \quad$ E.A. Para empresas importadoras o exportadoras}

T.M.R.R.I. $=27,53 \% \quad$ E.A. Para empresas netamente nacionales, en las cuales no tiene ninguna relación con el mercado externo es decir no exportan ni importan productos manufacturados o materias primas para su proceso.

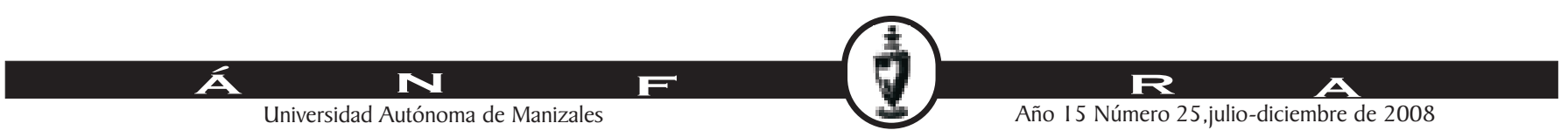


Tabla 2. Tasas para el cálculo de la T.M.R.R.I.

\begin{tabular}{|l|l|l|}
\hline Indicador & Abreviatura & Dato (2006) \\
\hline Rendimiento en bonos locales (TES) & $\mathrm{Rb}$ & $9,05 \% \mathrm{E} . \mathrm{A}$. \\
\hline $\begin{array}{l}\text { Tasa promedio en captación de } \\
\text { títulos CDT a 90 días del sistema } \\
\text { financiero Colombiano }\end{array}$ & DTF & $6,27 \% \mathrm{E} . \mathrm{A}$ \\
\hline $\begin{array}{l}\text { Tasa libre de riesgo de los bonos del } \\
\text { tesoro de los Estados Unidos } \\
\text { (treasury bonds) }\end{array}$ & $\mathrm{Rf}$ & $4,87 \% \mathrm{E} . \mathrm{A}$. \\
\hline Riesgo país (Indicador Embi) & $\mathrm{Rp}$ & $1,81 \% \mathrm{E} . \mathrm{A}$. \\
\hline $\begin{array}{l}\text { Riesgo de tipo de cambio } \\
\text { (devaluación revaluación) }\end{array}$ & $\mathrm{Rc}$ & $0,06 \% \mathrm{E} . \mathrm{A}$. \\
\hline Riesgo del inversionista & $\mathrm{Ri}$ & $8,39 \% \mathrm{E} . \mathrm{A}$. \\
\hline Riesgo sistémico del sector & $\mathrm{Beta}(\mathrm{B})$ & 0,72 \\
\hline
\end{tabular}

De acuerdo a la información anterior, el cálculo del costo de capital promedio ponderado (C.C.P.P.) para las 19 empresas más grandes del sector de alimentos para la ciudad de Manizales durante el año 2006 arrojó los siguientes resultados: Tabla 3

Un promedio del costo de capital para las 19 empresas más grandes del sector de alimentos de la ciudad de Manizales asciende al 21,31\% E. A.

Para el caso del costo de capital se tiene en cuenta un promedio para el estudio, debido a que la mayoría de los datos son homogéneos, es decir, presentan similitud en su resultado, a diferencia del cálculo de la rentabilidad los cuales no presentaron similitud. Estos datos presentan cierta uniformidad, debido a que las fuentes de financiación que generan el costo de capital son las mismas, aunque algunas empresas no hagan uso de las mismas fuentes.

\section{Cálculo Del Valor Económico Agregado (Eva)}

Para el cálculo del EVA, se debe definir con claridad las cuentas de los estados financieros necesarias para este cálculo:

Activo: Son todos los saldos contables que tiene una organización y se dividen en: activos corrientes, activos fijos y otros activos. Los activos corrientes son aquellos que son fácilmente convertibles en efectivo, es decir, son disponibles; algunos de ellos son: caja, bancos, cuentas por cobrar e inventarios; a estas cuentas también se les conoce como capital de trabajo ${ }^{8}$. Los activos fijos no están disponibles para la venta y con ellos se ejerce la actividad principal del negocio, algunos ejemplos son: la maquinaría, los muebles y enseres, los terrenos, los vehículos y el equipo de cómputo; también se encuentran los otros activos que no son de la actividad principal, como: gastos pagados por anticipado, los seguros y patentes.

De acuerdo a la anterior clasificación se debe tener en cuenta cuáles de estos activos son operativos, es decir, los que contribuyen a la generación de ingresos por ejercer la razón social de la empresa o negocio. Los activos corrientes y los activos fijos contribuyen a esta labor, más específicamente el disponible? ,: los bancos, las cuentas por cobrar y los inventarios y dentro de los activos fijos, que también son operativos tenemos el neto de estos. De acuerdo a lo anterior y para efectos del cálculo de la
8 Capital de trabajo, recursos monetarios necesarios para la correcta operación del negocio y poder ejercer de forma adecuada la actividad principal a la cual se dedica la empresa.

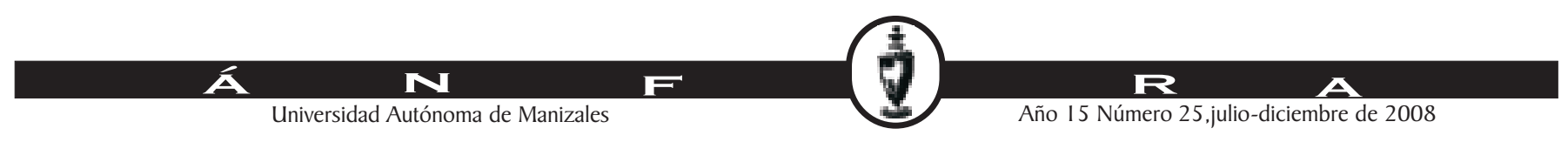


Tabla 3. Costo del capital promedio ponderado para las empresas de sector de alimentos de la ciudad de Manizales.

\begin{tabular}{|c|c|}
\hline \multicolumn{2}{|c|}{$\begin{array}{l}\text { CALCULO DEL COSTO DEL CAPITAL PROMEDIO PONDERADO } \\
\text { C.C.P.P. }\end{array}$} \\
\hline EMPRESA & C.C.P.P. \\
\hline INDUSTRIAS NORMANDY S.A. & $23,33 \%$ \\
\hline COLOMBIANA DE DESHIDRATADOS S.A. & $26,94 \%$ \\
\hline PANADERIA LA VICTORIA S.A. & $26,78 \%$ \\
\hline CENRAL DE SACRIFICIO MANIZALES S.A. & $20,64 \%$ \\
\hline C.I. SUPER DE ALIMENTOS S.A. & $15,41 \%$ \\
\hline SOCIEDAD PRODUCTOS LACTEOS DE CALDAS & $23,08 \%$ \\
\hline INDIANA S.A. & $27,57 \%$ \\
\hline CENTRAL LECHERA DE MANIZALES S.A. & $21,49 \%$ \\
\hline PRODUCTORA DE GELATINA S.A. & $22,04 \%$ \\
\hline INDUSTRIAS DE ALIMENTOS GRANSOLI Y CIA. & $22,98 \%$ \\
\hline ALTOPASTI S.A. & $7,08 \%$ \\
\hline H.M. Y CIA. S. EN C. A. & $27,23 \%$ \\
\hline SUZETTE S.A. & $8,64 \%$ \\
\hline ARISTIZABAL ARANGO Y CIA. S. EN C. A. & $7,66 \%$ \\
\hline CAFÉ NEVADO LTDA. & $27,07 \%$ \\
\hline INCOLFEC S.A. & $21,11 \%$ \\
\hline DELI APA S.A. & $27,22 \%$ \\
\hline FOODEX S.A. & $22,28 \%$ \\
\hline DESCAFEINADORA COLOMBIANA S.A. & $26,30 \%$ \\
\hline
\end{tabular}

rentabilidad del activo, tendremos en cuenta solamente los activos fijos y el capital de trabajo.

Pasivo: Son todos los compromisos económicos que tiene la organización con terceras personas, es decir con los proveedores, las entidades financieras, los empleados y el estado; estas obligaciones se dividen en corto y largo plazo. Por lo general, a nivel contable, se consideran de corto plazo las obligaciones cuya duración es menor a un año y de largo plazo aquellas que superan el año; para la realización del cálculo del valor económico agregado se deben tener en cuenta aquellos pasivos que son generadores de costo, es decir, aquellos que por la tasa de interés de la cuenta con el tercero tiene un costo de financiación que hace que la rentabilidad de la empresa disminuya por el solo hecho de hacer uso de unos recursos que no son propios y que hacen que se tenga el deber de pagar. Dentro de los pasivos, algunas obligaciones generadoras del costo son: las financieras, tanto de largo como de corto plazo, algunas obligaciones laborales, la emisión de bonos y otros títulos valores.

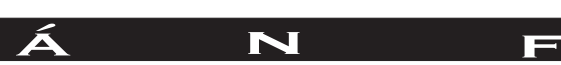
Universidad Autónoma de Manizales 
Hay otros pasivos que, al contrario, no son generadores de costo de financiación en la medida en que la empresa los cancela a su debido tiempo como son las obligaciones con el estado a través de los impuestos como el de Renta el cual se cancela de forma anual y se genera de forma mensual (en la mayoría de organizaciones) lo que hace que el costo de financiación sea $0 \%$; también los proveedores en la medida que las obligaciones sean canceladas en el tiempo pactado no generan costo alguno.

Patrimonio: Son las obligaciones que tiene la empresa con sus dueños o accionistas y para efectos de su estudio, el costo que genera la utilización del patrimonio está determinada por la tasa que estarían dispuestos a cobrar los socios o accionistas por tener su dinero en la organización, el valor de este costo se puede determinar a través de la tasa mínima de rendimiento requerido por el inversionista (T.M.R.R.I.)

Tanto los pasivos como el patrimonio son necesarios en el estudio, con el objeto de determinar el costo de capital promedio ponderado.

Una vez aplicada la fórmula se obtienen los siguientes resultados del EVA por empresa:

Tabla 4. Cálculo del Valor Económico Agregado (EVA) para las empresas del sector de alimentos de la ciudad de Manizales en términos porcentuales y monetarios correspondiente al periodo 2006. Realizado por el autor.

\begin{tabular}{|l|l|}
\hline INDUSTRIAS NORMANDY S.A. & \\
\hline Rentabilidad del Activo & $9,33 \%$ \\
\hline Costo de Capital Promedio Ponderado & $\mathbf{2 3 , 3 3 \%}$ \\
\hline Activos operativos & $\mathbf{3 . 6 7 4 . 2 0 6}$ \\
\hline Valor Económico Agregado \% & $\mathbf{- 1 4 , 0 0 \%}$ \\
\hline Valor Económico Agregado \$ & $\mathbf{- 5 1 4 . 3 8 9}$ \\
\hline
\end{tabular}

\begin{tabular}{|l|l|}
\hline \multicolumn{2}{|l|}{ COLOMBIANA DE DESHIDRTADOS S.A. } \\
\hline Rentabilidad del Activo & $9,16 \%$ \\
\hline Costo de Capital Promedio Ponderado & $\mathbf{2 6 , 9 4 \%}$ \\
\hline Activos operativos & $\mathbf{7 8 6 . 7 6 4}$ \\
\hline Valor Económico Agregado \% & $\mathbf{- 1 7 , 7 8 \%}$ \\
\hline Valor Económico Agregado \$ & $\mathbf{- 1 3 9 . 8 8 7}$ \\
\hline
\end{tabular}

\begin{tabular}{|l|l|}
\hline \multicolumn{2}{|l|}{ PANADERIA LA VICTORIA } \\
\hline Rentabilidad del Activo & $\mathbf{8 , 5 4 \%}$ \\
\hline Costo de Capital Promedio Ponderado & $\mathbf{2 6 , 7 8 \%}$ \\
\hline Activos operativos & $\mathbf{2 . 1 1 0 . 5 5 2}$ \\
\hline Valor Económico Agregado \% & $\mathbf{- 1 8 , 2 4 \%}$ \\
\hline Valor Económico Agregado \$ & $\mathbf{- 3 8 4 . 9 6 5}$ \\
\hline
\end{tabular}

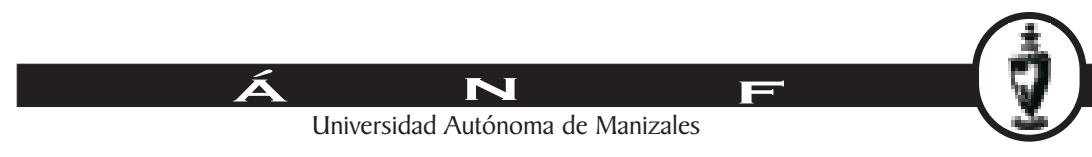


CENTRAL DE SACRIFICIO MANIZALES S.A.

Rentabilidad del Activo

$12,12 \%$

Costo de Capital Promedio Ponderado

$20,64 \%$

Activos operativos

4.960 .963

Valor Económico Agregado \%

$-8,52 \%$

Valor Económico Agregado \$

$-422.674$

C.I. SUPER DE ALIMENTOS S.A.

Rentabilidad del Activo

$7,02 \%$

Costo de Capital Promedio Ponderado

$15,41 \%$

Activos operativos

65.675 .338

Valor Económico Agregado \%

$-8,39 \%$

Valor Económico Agregado \$

$-5.510 .161$

\section{SOCIEDAD PRODUCTOS LACTEOS DE CALDAS}

Rentabilidad del Activo

$0,57 \%$

Costo de Capital Promedio Ponderado

$23,08 \%$

Activos operativos

368.812

Valor Económico Agregado \%

$-22,51 \%$

Valor Económico Agregado \$

$-83.020$

INDIANA S.A.

Rentabilidad del Activo $-0,19 \%$

Costo de Capital Promedio Ponderado

$27,57 \%$

Activos operativos

237.484

Valor Económico Agregado \%

$-27,76 \%$

Valor Económico Agrega do \$

$-65.926$

CENTRAL LECHERA DE MANIZALES S.A.

Rentabilidad del Activo $3,77 \%$

Costo de Capital Promedio Ponderado

Activos operativos

$21,49 \%$

Valor Económico Agregado \%

9.808 .213

Valor Económico Agregado \$

$-17,72 \%$

$-1.738 .015$ 
PRODUCTORA DE GELATINA S.A.

Rentabilidad del Activo $4,60 \%$

Costo de Capital Promedio Ponderado $22,04 \%$

Activos operativos 42.858.106

Valor Económico Agregado \% $-17,44 \%$

Valor Económico Agregado \$

$-7.474 .454$

INDUSTRIAS DE ALIMENTOS GRANSOLI Y CIA.

Rentabilidad del Activo

Costo de Capital Promedio Ponderado

Activos operativos

Valor Económico Agregado \%

Valor Económico Agregado \$ $1,09 \%$ $22,98 \%$ 595.957 $-21,89 \%$ $-130.455$

\begin{tabular}{|l|l|}
\hline \multicolumn{2}{|l|}{ AlTOPASTI S.A. } \\
\hline Rentabilidad del Activo & $-13,07 \%$ \\
\hline Costo de Capital Promedio Ponderado & $7,08 \%$ \\
\hline Activos operativos & $\mathbf{1 8 5 . 9 7 6}$ \\
\hline Valor Económico Agregado \% & $\mathbf{- 2 0 , 1 5 \%}$ \\
\hline Valor Económico Agregado \$ & $\mathbf{- 3 7 . 4 7 4}$ \\
\hline
\end{tabular}

HM Y CIA. S EN C. A.

Rentabilidad del Activo

$1,40 \%$

Costo de Capital Promedio Ponderado

$27,23 \%$

Activos operativos

4.523 .811

Valor Económico Agregado \%

$-25,83 \%$

Valor Económico Agregado \$

$-1.168 .500$

SUZETTE S.A.

Rentabilidad del Activo

Costo de Capital Promedio Ponderado

Activos operativos

Valor Económico Agregado \%

Valor Económico Agregado \$

$1,49 \%$

$8,64 \%$

176.316

$-7,15 \%$

$-12.607$ 
ARISTIZABAL ARANGO Y CIA. S. EN C. A.

Rentabilidad del Activo

$-183,81 \%$

Costo de Capital Promedio Ponderado

$7,66 \%$

Activos operativos

812.555

Valor Económico Agregado \%

$-191,47 \%$

Valor Económico Agregado \$

\section{CAFÉ NEVADO LTDA.}

Rentabilidad del Activo

$-9,52 \%$

Costo de Capital Promedio Ponderado

$27,07 \%$

Activos operativos

140.759

Valor Económico Agregado \%

$-36,59 \%$

Valor Económico Agregado \$

$-51.504$

INCOLFEC S.A.

Rentabilidad del Activo

$3,81 \%$

Costo de Capital Promedio Ponderado

$21,11 \%$

Activos operativos

Valor Económico Agregado \%

1.706 .185

Valor Económico Agregado \$

$-17,30 \%$

$-295.170$

\section{DELI APA S.A.}

Rentabilidad del Activo $8,99 \%$

Costo de Capital Promedio Ponderado

$27,22 \%$

Activos operativos

568.293

Valor Económico Agregado \%

$-18,23 \%$

Valor Económico Agregado \$

$-103.600$

\section{FOODEX S.A.}

Rentabilidad del Activo

Costo de Capital Promedio Ponderado

$3,23 \%$

Activos operativos

$22,28 \%$

Valor Económico Agregado \%

37.449 .552

Valor Económico Agregado \$

$-19,05 \%$

$-7.134 .140$ 


\begin{tabular}{|l|l|}
\hline \multicolumn{2}{|l|}{ DESCAFEINADORA COLOMBIANA S.A. } \\
\hline Rentabilidad del Activo & $-0,46 \%$ \\
\hline Costo de Capital Promedio Ponderado & $\mathbf{2 6 , 3 0 \%}$ \\
\hline Activos operativos & $\mathbf{2 8 . 8 9 6 . 6 2 5}$ \\
\hline Valor Económico Agregado \% & $\mathbf{- 2 6 , 7 6 \%}$ \\
\hline Valor Económico Agregado \$ & $\mathbf{- 7 . 7 3 2 . 7 3 7}$ \\
\hline
\end{tabular}

Al igual que en el caso del cálculo de la rentabilidad no se obtiene un promedio del Valor Económico Agregado para las empresas de alimentos de la ciudad de Manizales, debido a que este indicador está ligado de forma directa con la rentabilidad del activo lo que hace que sus resultados no sean homogéneos, por lo tanto con un promedio no se pueden obtener conclusiones.

Adicionalmente, en la actualidad no hay estadísticas sectoriales sobre la rentabilidad y generación de valor, lo que dificulta el proceso de conclusiones, pero es importante destacar que esta información para el año 2006 es la base para nuevos estudios de periodos posteriores para comenzar a tener información sectorial para determinar la eficiencia de este en el mercado.

\section{Conclusiones}

- En el cálculo del costo del capital promedio ponderado se pudo observar que 16 empresas de las 19 estudiadas, en su mayoría, se financian en mayor proporción con patrimonio (recursos propios) que con pasivos (recursos de terceros), lo que causa que el costo de capital se incremente debido a que el riesgo es mayor en la medida que los recursos están invertidos en un solo negocio, contrario a lo que ocurre con los recursos de terceros los cuales se encuentran diversificados lo que hace que el riesgo disminuya y causa un costo de financiación menor. Muchos empresarios tienen la creencia que la financiación con recursos propios es a costo cero, debido a que no tiene en cuenta el riesgo que se corre en la inversión.

- Los empresarios miden su rentabilidad a partir de las utilidades que se generan al interior de la organización, siendo este un parámetro de corto plazo, cuando la rentabilidad puede enfocarse a largo plazo pero el empresario no tiene eso en cuenta, debido a que lo importante para este tipo de empresario, como se presenta en la mayoría de los casos, es el resultado obtenido al final del periodo (utilidad neta) y no los resultados que se puedan obtener en el largo plazo y el Valor Económico Agregado es un indicador de largo plazo

- $\quad$ Las principales empresas del sector de alimentos de la ciudad de Manizales son poco rentables en su actividad principal (rentabilidad del activo) esto debido a varios factores como son: a) El 50\% de las empresas estudiadas presenta un capital de trabajo demasiado alto con respecto a su propiedad planta y equipo lo que muestra que hay un problema de rotación en estas organizaciones, debido a que los saldos de estas cuentas debe ser bajo en la medida que se haga buen uso de los recursos como son el efectivo, las cuentas por cobrar y los inventarios b) Algunas de las empresas estudiadas tiene altos niveles de sus saldos de activos fijos, lo que hace deducir que hay activos fijos operativos no utilizados de forma

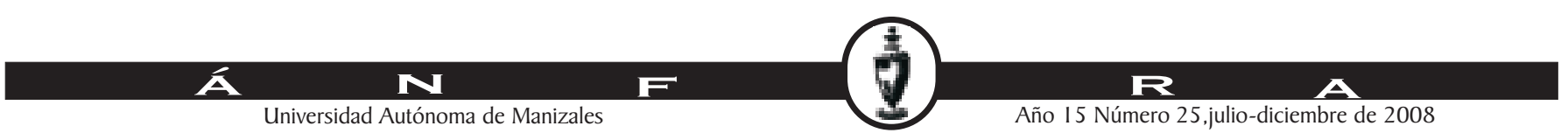


adecuada debido a las bajas utilidades que se dan con respecto a la gran inversión en activos de capital.

- $\quad$ Ninguna de las empresas objeto de estudio es generadora de valor, por lo contrario todas son destructoras de valor y esto se debe principalmente a la baja rentabilidad de la actividad principal como el alto costo de capital en que incurren al realizar su financiación, el costo de capital promedio para las empresas estudiadas arrojó un $21,31 \%$ E.A. mientras que ninguna mostró una rentabilidad que superara este costo siendo la empresa más rentable la Central de Sacrificio de Manizales la cual alcanzó una rentabilidad del 12\% E.A.

\section{Recomendaciones}

- Los empresarios del sector de alimentos de Manizales deben vislumbrar sus negocios hacía el largo plazo, es decir, deben crear las estrategias para el logro de una mayor rentabilidad producto de sus operaciones.

- Lastimosamente en nuestro País hay una cultura de generación de utilidades, estrategia del corto plazo, esto debe cambiar debido a que la rentabilidad al ser una estrategia del largo plazo hace que la competitividad de estas organizaciones aumente, debido a que se crean las estrategias para poder actuar de otra forma más eficientes en mercados más competitivos.

- Realizar un seguimiento de la generación o destrucción de valor para las empresas objeto de estudio, con el objeto de poder determinar nuevas conclusiones sobre el empresario del sector, debido a que si se hace un seguimiento, seguramente se hace un refinamiento de la información, para poder aplicar estas nuevas conclusiones a otros sectores y a los empresario brindarle mejor y mayor información para que apunten a un sector y empresa más competitiva.

- Continuar con el proceso investigativo para periodos posteriores para poder determinar de esta manera una verdadera evolución del sector, como también para poder observar el grado de competitividad que estas van adquiriendo a medida que pasa el tiempo.

- Hacer conocer los resultados de esta investigación a los empresarios de las organizaciones objeto del estudio, con el propósito de que comiencen a tomar decisiones de largo plazo enfocadas hacía la generación de valor y poder así obtener información más detallada en futuros procesos investigativos para el mejoramiento de la información.

- $\quad$ Aunque en la actualidad no existe un marco legal que exija a las empresas realizar un análisis más profundo de sus resultados y que estos se enfoquen a la generación de valor, crecimiento organizacional o mayor competitividad del sector para que apunten a mercados más grandes o de índole internacional, el estudio de los resultados debería convertirse en una cultura organizacional que apunte al logro de estos objetivos para el mejoramiento del sector y de la competitividad empresarial.

- Con nuevos estudios se recomienda que los gremios, como ACOPI, sean líderes en la implementación de estrategias para que los empresarios del sector puedan tener

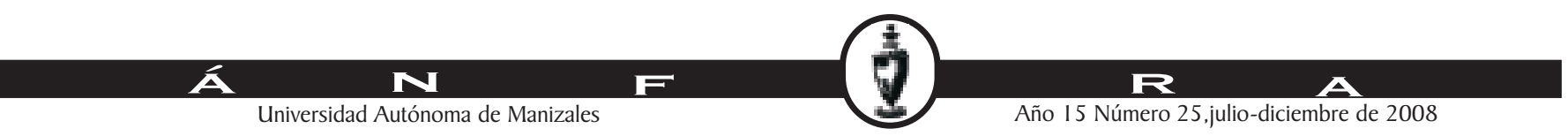


más información y capacitación sobre el tema para la implementación de nuevos métodos de análisis enfocados al logro del valor empresarial.

- Poder contar con la información más rápidamente debido a que hasta la fecha solamente se pudo obtener estados financieros con corte al año 2006 y para poder realizar los estudios se hace necesario contar con los estados financieros los cuales aún no están disponibles, es decir la información es suministrada con 2 años de vencimiento aproximadamente.

\section{Bibliografia}

AMAT, Oriol. Valor Económico agregado. Un enfoque para optimizar la gestión empresarial, motivar a los empleados y crear valor. Grupo editorial Norma. Año 1999. Edición 1.

ÁLVAREZ, Alberto. Matemáticas financieras. Segunda edición. Editorial Mc Graw Hill. 1999.

ANDERSEN, Arthur. Diccionario de economía y negocios. Editorial espasa. 1999.

BACA CURREA, Guillermo. Ingeniería Económica. Editorial Fondo Educativo Panamericano. Sexta Edición. 2000.

COPELAND, Thomas E. Finanzas en administración, novena edición año 1992. Mc Graw Hill.

ESCOBAR ARI AS, Gabriel Eduardo. Finanzas Básicas. SIC editorial. 2003.

ESTUPIÑÁN GAITÁN, Rodrigo. Análisis financiero y de gestión. Ecoe ediciones. 2003.

GARCÍA, Oscar León. Valoración de empresas, gerencia del valor y EVA. Impreso por Digital Express en la ciudad de Medellín año 2003.

GARCÍA, oscar León. Administración financiera, fundamentos aplicaciones. Impreso en Medellín. Tercera edición.

GORDON J, Alexander. Fundamentos de inversiones. Teoría y práctica. Editorial Pearson Educación. 2003.

KAPLAN, Robert S. El cuadro del mando integral the balanced scorecard. Gestión 2000. Barcelona 1997.

LA REPUBLICA, diccionario de términos financieros y bursátiles. Editorial el Globo año 1999.

MEZA OROZCO, J honny de Jesús. Matemáticas financieras aplicadas. Ecoe ediciones. Primera edición, Valledupar 2002.

OSVALDO BRAND, Salvador. Diccionario de economía. Plaza y J anes Editores. Séptima edición año 2003.

STERN, J oel M. y Shiely, J hon S. El Desafío del EVA. Como implementar el cambio en la organización. Grupo Editorial Norma. 2002.

TERREL, J ones y TERREL, Werner. Introducción a la contabilidad financiera. Editorial Prentice Hall. 2001.

VAN HORNE, James. Administración financiera. Prentice Hall. Año 1997.

VÉLEZ PAREJA, Ignacio. Decisiones bajo riesgo e incertidumbre. Grupo Editorial Norma. 2003.

Internet:

http://www.wikilearning.com/el_valor_economico_agregado-wkc-13182.htm

http://www.12manage.com/methods_eva_es.html

http://www.kreston.es/stern-stewart.htm 
http://www.businesscol.com/productos/glosarios/economico/glosario_economia_a.h tml

http: //gerenciaactual. blogspot.com/2007/07/el-eva-o-valor-econmicoagregado.html

http://www.grupobancolombia.com/home/index.asp

http://www.portafolio.com.co/

http://pagos.semana.com/promociones/dinero.html

http://www.superfinanciera.gov.co

http://www.supervalores.gov.co

http://www.supersociedades.gov.co

http://www.larepublica.com.co

\section{A N}

